\title{
Infrared activity in elemental crystals
}

\author{
Richard Zallen \\ Department of Physics, Virginia Tech, Blacksburg, Virginia 24061 \\ Richard M. Martin and Vincent Natoli \\ Department of Physics, University of Illinois at Urbana-Champaign, Urbana, Illinois 61821
}

(Received 7 October 1993)

\begin{abstract}
In a previous paper, Zallen [Phys. Rev. 173, 824 (1968)] reported a group-theoretical analysis of the competition between unit-cell complexity and crystal symmetry in determining the presence or absence of infrared-active phonons in an elemental crystal. Here we correct an error in that paper's treatment of certain hexagonal space groups. Our results modify the minimum-complexity condition for infrared activity: For 228 of the 230 space groups, a necessary and sufficient condition for the existence of symmetry-allowed infrared-active modes in an elemental crystal is the presence of three or more atoms in the primitive unit cell. The two exceptional space groups are $P 6 / \mathrm{mmm}\left(D_{6 h}^{1}\right)$ and $P 6_{3} / \mathrm{mmc}\left(D_{6 h}^{4}\right)$; for each of these symmetries, there exists one structure with four atoms per cell and no infrared modes. The $P 6_{3} / m m c$ structure includes, as special cases, Lonsdaleite (or "wurtzite silicon") as well as a $c$-axisaligned hcp arrangement of diatomic molecules which is relevant to models of solid molecular hydrogen at high pressure.
\end{abstract}

Crystals composed of a single atomic species can exhibit strong one-phonon infrared absorption lines by the mechanism of displacement-induced charge redistribution (“dynamic charge"). ${ }^{1}$ For an elemental crystal to exhibit such first-order infrared activity, the crystal symmetry, and unit-cell structure must admit symmetry-allowed infrared-active vibrational modes. Reference 2 (henceforth Z68) presented a detailed group-theoretical analysis of the competition between unit-cell complexity and crystal symmetry which determines the presence or absence of infrared activity in an elemental crystal. A general conclusion of Z68 was the existence of a minimumcomplexity condition for the occurrence of infraredactive phonons in an elemental crystal. The purpose of this Brief Report is to rectify an error in Z68 and to present the correct statement of that minimumcomplexity condition, which illuminates the connection between crystal structure and photon-phonon coupling. The statement given in Z68 is expressed below in lines (1) and (2). Let $s$ denote the number of primitive-cell atoms and $n_{v}^{\text {ir }}$ the number of infrared (ir) eigenfrequencies (number of reststrahlen bands). Then

$$
\begin{aligned}
& n_{v}^{\mathrm{ir}}=0 \leftrightarrow s=1 \text { or } s=2, \\
& n_{v}^{\mathrm{ir}} \geq 1 \leftrightarrow s \geq 3 .
\end{aligned}
$$

Line (1) states that $s \geq 3$ is a necessary condition for infrared activity, and (2) asserts that it is also a sufficient condition. But here we show that (2) does not hold for two of the 230 space groups.

Statements (1) and (2) agree with a wide variety of results, including: the absence of infrared phonons in $\mathrm{Si}$, $\mathrm{Ge}$, and diamond ${ }^{3}(s=2)$ and rhombohedral $\mathrm{As}, \mathrm{Sb}$, and
Bi $(s=2)$; and the observation of infrared phonons in trigonal selenium ${ }^{4}$ and tellurium ${ }^{5}(s=3)$; graphite $^{6}(s=4)$; orthorhombic chlorine, bromine, and iodine ${ }^{7}(s=4)$; cubic nitrogen ${ }^{8}(s=8)$; orthorhombic sulfur ${ }^{9}$ and monoclinic selenium ${ }^{10}(s=32)$, and fullerene ${ }^{11}(s=60)$. However, in the course of considering candidate structures for the high-pressure molecular phase of solid hydrogen, ${ }^{12}$ we uncovered an exception to (2) which prompted a reexamination of Z68 and the discovery of an error in the treatment of six hexagonal space groups. Here we remove that error and show that (2) is valid with the exception of two special hexagonal structures with $s=4$.

We adopt the same notation as in Z68: $G$ is the crystal factor group, of order $g ; \alpha$ is an operation belonging to $G$; $\Gamma_{i}$ is an irreducible representation (IR) of $G ; P$ is the three-dimensional polar-vector representation of $G ; \Gamma_{p}$ is a $\Gamma_{i}$ occurring in the reduction of $P$ (a vector IR), $\left\{\Gamma_{p}\right\} \subset\left\{\Gamma_{i}\right\}$, and $h$ is the number of $\Gamma_{p}$ (e.g., 3 for orthorhombic groups, 2 for hexagonal, 1 for cubic); $\chi^{P}(\alpha)$ is the character for operation $\alpha$ in representation $P$; $\Gamma$ is the $3 s$-dimensional representation of $G$ generated by the displacements of the $s$ atoms in the primitive cell; $s(\alpha)$ is the site-permutation character ${ }^{13}$ of $\alpha$, the number of sublattices (primitive-cell atomic positions) invariant under $\alpha$; $n^{\text {ir }}$ is the number of infrared-active eigenvibrations; and $n_{v}^{\text {ir }}$ is the number of infrared-active eigenfrequencies.

Relevant results derived in Z68 are briefly summarized here. For five low-symmetry crystal classes $(1,2, m, \overline{1}$, and $2 / m),{ }^{14}(1)$ and (2) are easily proved. For the remaining 27 crystal classes, the general expression for $n_{v}^{\text {ir }}$ takes the form

$$
n_{v}^{\text {ir }}=(1 / g) \sum_{\alpha}[s(\alpha)-1]\left|\chi^{P}(\alpha)\right|^{2} .
$$


Since each $\Gamma_{p}$ occurs just once in the reduction of $P$ for each of these 27 classes, it follows that

$$
\sum_{\alpha}\left|\chi^{P}(\alpha)\right|^{2}=g h \text {. }
$$

Since $s(1)=s$ and $\left|\chi^{P}(1)\right|^{2}=9$, we can then rewrite Eq. (3) as

$$
g n \underset{v}{\text { ir }}=9 s-g h+\sum_{\alpha \neq 1} s(\alpha)\left|\chi^{P}(\alpha)\right|^{2}
$$

Neglecting the (non-negative) sum in (5) yields a lower limit for $n_{v}^{\text {ir }}$

$$
g n_{v}^{\text {ir }} \geq 9 s-g h .
$$

This inequality succinctly captures the competition between unit-cell complexity (represented by $9 s$ ) and the constraints imposed by symmetry (represented by $g h$ ) in determining whether or not infrared-active modes appear.

For 23 of the 27 remaining crystal classes, $g h$ is no larger than 24 , so that the right side of $(6)$ is positive when $s$ is three or more. This establishes (2) for these 23 classes and leaves only four high-symmetry classes for which $g h$ exceeds 24 . These four are $m 3 m(g=48, h=1)$, $6 / \mathrm{mmm} \quad(g=24, h=2), \quad 4 / \mathrm{mmm}(g=16, h=2), \quad$ and $6 / m(g=12, h=3)$.

For the four high-symmetry classes, the method adopted in Z68 was equivalent to considering the effect on inequality (6), for specific $s$ values, of contributions arising from the sum in (5). Such contributions arise when $s(\alpha)$ is nonzero [except for $\alpha=1$, which is absent from the sum, and $\alpha=3$ and $\alpha=\overline{3}$, for which $\chi^{P}(\alpha)$ is zero]. The error in Z68 occurred in omitting, for $s=4$, two operations from the list of those for which $s(\alpha)=0$ is possible when $s=4$; these are $\alpha=6$ and $\alpha=\overline{6}$. This omission does not affect the results (the validity of the sufficiency of $s \geq 3$ for infrared activity) for classes $m 3 m$ and $4 / \mathrm{mmm}$, since these groups lack 6 and $\overline{6}$ hexad axes. However, it does affect the results for classes $6 / \mathrm{mmm}$ and $6 / \mathrm{m} .{ }^{15}$ For both classes, it leaves open the possibility that $n_{v}^{\text {ir }}=0$ when $s=4$.

There are two space groups in class $6 / \mathrm{m}$ and four in class $6 / \mathrm{mmm}^{14}$ We now examine the possible $s=4$ structures for these six space groups. ${ }^{16}$ Since we are looking for possible $n_{v}^{\text {ir }}=0$ structures, we need to examine structures based on a single set of multiplicity-four equivalent positions, because $n_{v}^{\text {ir }} \geq 1$ if more than one set of equivalent sites is occupied. ${ }^{17}$ The two $6 / \mathrm{m}$ space groups are $P 6 / m\left(C_{6 h}^{1}\right.$, number 175) and $P 6_{3} / m\left(C_{6 h}^{2}\right.$, 176). For both groups, examination of the symmetries reveals that the structures which result from the occupation of a single set of multiplicity-four sites have $6 / \mathrm{mmm}$, not $6 / \mathrm{m}$, symmetry. Thus there are no $s=4$ $6 / m$ structures based on a single set of equivalent sites.

Only class $6 / \mathrm{mmm}$ remains to consider, and it is here that we find the two exceptional structures, for which (2) does not hold. The four space groups with maximal hexagonal symmetry $\left(6 / \mathrm{mmm}\right.$, or $\left.D_{6 h}\right)$ are numbers 191-194 in the standard listing: ${ }^{14} P 6 / \mathrm{mmm}\left(D_{6 h}^{1}\right)$, $P 6 / m c c\left(D_{6 h}^{2}\right), P 6_{3} / m c m\left(D_{6 h}^{3}\right)$, and $P 6_{3} / m m c\left(D_{6 h}^{4}\right)$.
For $D_{6 h}^{2}$ and $D_{6 h}^{3}$, each multiplicity-four set of sites, if solely occupied, yields an $s=2 D_{6 h}^{1}$ structure (with a halved $c$-axis primitive-cell height relative to the initial $D_{6 h}^{2}$ or $D_{6 h}^{3}$ cell). But both $D_{6 h}^{1}$ and $D_{6 h}^{4}$ each do have one structure based on a quartet of equivalent sites with $3 m$ symmetry. And for both of these $s=4$ structures, $n_{v}^{\text {ir }}=0$; there are no symmetry-allowed infrared-active modes.

The two special structures are shown in Fig. 1 and specified in the first two rows of Table I. Three other elemental structures are included for comparison in Table I. Structure A, the $D_{6 h}^{1} s=4$ structure, does not, to our knowledge, occur for any elemental solid. Structure B, the $D_{6 h}^{4} s=4$ structure, is referred to in the table as $c$ aligned hcp $X_{2}$, since this structure is closely connected
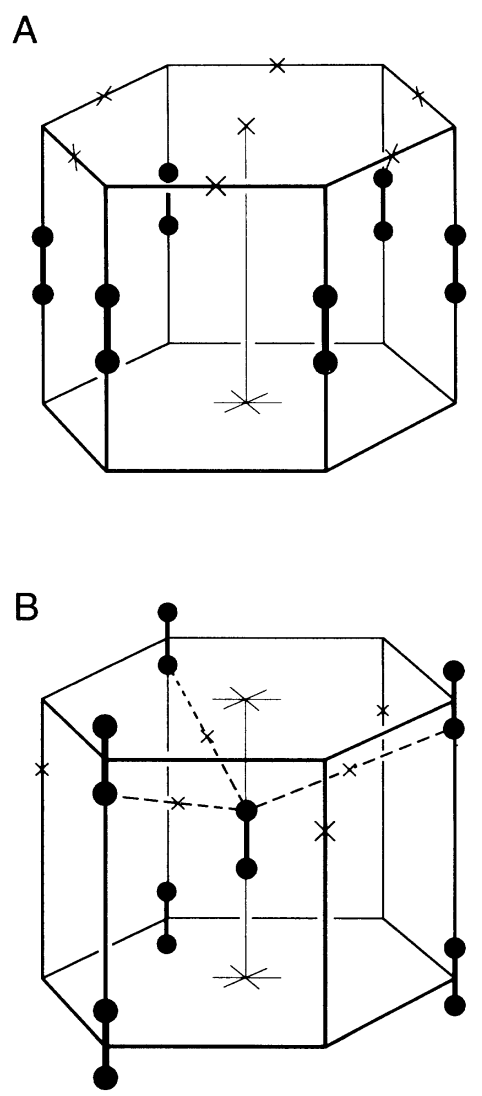

FIG. 1. The two infrared-inactive elemental crystal structures with more than two atoms per primitive cell. Both are hexagonal structures (A's symmetry is $P 6 / \mathrm{mmm}$, B's is $\left.P 6_{3} / m m c\right)$ with four-atom cells. The $c / a$ ratio is arbitrary. Heavy dots denote atomic sites, crosses indicate inversion centers in one horizontal plane. The heavy vertical bonds, all equal in length, constitute an internal degree of freedom for each structure. For a special value of this bond length and the ideal $c / a$ ratio, B becomes the Lonsdaleite (or "wurtzite silicon") structure. The symmetry remains $P 6_{3} / m m c$ but the coordination becomes tetrahedral, with the dashed bonds equal in length to the vertical bond. 
TABLE I. Comparison of the two exceptional $s=4$ hexagonal structures (A and B), for which infrared activity is symmetry forbidden, with other elemental crystals. Lonsdaleite (or "wurtzite Si") is a special case of B corresponding to $d=3 / 16$ and $(c / a)=1.633$. The site-permutation characters $s(\alpha)$ are defined in the text.

\begin{tabular}{|c|c|c|c|c|c|c|c|}
\hline \multirow[b]{2}{*}{ Structure } & \multirow{2}{*}{$\begin{array}{l}\text { Space } \\
\text { group }\end{array}$} & \multirow[b]{2}{*}{$s$} & \multirow[b]{2}{*}{ Atomic positions ${ }^{\mathrm{a}}$} & \multirow{2}{*}{$\begin{array}{c}\text { Site } \\
\text { symm. }\end{array}$} & \multirow{2}{*}{$\begin{array}{l}\text { Symmetry-operation classes } \\
\text { for which } s(\alpha) \text { is nonzero }\end{array}$} & \multicolumn{2}{|c|}{ Infrared } \\
\hline & & & & & & $n_{v}^{\mathrm{ir}}$ & $n^{\text {ir }}$ \\
\hline A. Fig. $1 \mathrm{~A}$ & $P 6 / \mathrm{mmm}$ & 4 & $\left(\frac{1}{3}, \frac{2}{3}, \pm d\right)\left(\frac{2}{3}, \frac{1}{3}, \pm d\right)$ & $3 m$ & $s(\alpha)=4$ for $\alpha=1,3, \overline{2}^{\prime}$ & 0 & 0 \\
\hline B. $c$-aligned hcp $X_{2}$ & $P 6_{3} / m m c$ & 4 & $\left(\frac{1}{3}, \frac{2}{3}, \frac{1}{4} \pm d\right)\left(\frac{2}{3}, \frac{1}{3}, \frac{3}{4} \pm d\right)$ & $3 m$ & $s(\alpha)=4$ for $\alpha=1,3, \overline{2}^{\prime}$ & 0 & 0 \\
\hline C. Graphite & $P 6_{3} / m m c$ & 4 & $\begin{array}{l}\left(0,0, \frac{1}{4}\right)\left(\frac{1}{3}, \frac{2}{3}, \frac{1}{4}\right) \\
\left(0,0, \frac{3}{4}\right)\left(\frac{2}{3}, \frac{1}{3}, \frac{3}{4}\right)\end{array}$ & $\overline{6} m 2$ & $\begin{array}{l}s(\alpha)=4 \text { for } \\
\alpha=1,3,2^{\prime \prime}, \overline{6}, \overline{2}, \overline{2}^{\prime}\end{array}$ & 2 & 3 \\
\hline D. $\gamma-N_{2}$ & $P 4_{2} / m n m$ & 4 & $\pm(x, x, 0) \pm\left(\frac{1}{2}+x, \frac{1}{2}-x, \frac{1}{2}\right)$ & $m .2 m$ & $\begin{array}{l}s(\alpha)=4 \text { for } \alpha=1, \overline{2} \\
s(\alpha)=2 \text { for } \alpha=2^{\prime \prime}, \overline{2}^{\prime \prime}\end{array}$ & 1 & 2 \\
\hline E. Off-axis hcp $\mathrm{H}_{2}$ & $P 2_{1} / c$ & 8 & Not special & 1 & $s(\alpha)=8$ for $\alpha=1$ & 9 & 9 \\
\hline
\end{tabular}

${ }^{a}$ Coordinates are referred to hexagonal lattice vectors for $A, B$, and $C$; tetragonal lattice vectors for $D$ (as in $\operatorname{Ref}$. 14).

to structures observed for diatomic molecules (including $\mathrm{H}_{2}, \mathrm{D}_{2}$, and $\mathrm{N}_{2}$ ) (Ref. 18) arranged in hexagonal close packing. In both $A$ and $B$, the four unit-cell atoms occur in two pairs, each pair aligned parallel to the hexad axes. Both structures possess one internal degree of freedom, the $c$-axis separation within each pair (the quantity $2 d$ in Table I; for $c$-aligned hcp $X_{2}$, this is the $X$ - $X$ bond length). The Lonsdaleite (or "wurtzite silicon") (Ref. 19) form of silicon ${ }^{20}$ and carbon ${ }^{21}$ corresponds to a special value of this separation. No new symmetry arises in the special case (of $B$ ) corresponding to Lonsdaleite $(d / c=3 / 16$ and $c / a=1.633)$, but each atom is now tetrahedrally coordinated.

For both $A$ and $B, \Gamma$ is given by $A_{1 g}+B_{1 g}+E_{1 g}+E_{2 g}+A_{2 u}+B_{2 u}+E_{1 u}+E_{2 u}$. Once the acoustic modes $\left(A_{2 u}+E_{1 u}\right)$ are accounted for, no $\Gamma_{p}$ 's remain for the optical modes, so none can be infrared active. As seen in Table I, graphite has $s=4$ and belongs to the same group as $B$, yet possesses infraredactive modes. (For graphite, $\Gamma$ is $2 B_{1 g}+2 E_{2 g}+2 A_{2 u}$ $+2 E_{1 u}$.) This illustrates the importance of the site- permutation characters $s(\alpha)$, which account for the difference in infrared activity.

Structures D and E in Table I are included since, like $B$, they are relevant to possible high-pressure phases of hydrogen. ${ }^{12,22} \mathrm{D}$ is the structure of $\gamma$-nitrogen and is a herringbone structure with two orthogonally oriented molecules per tetragonal primitive cell. ${ }^{18} \mathrm{E}$ is a monoclinic $s=8$ structure which can be derived from B by tilting molecules off the $c$ axis. ${ }^{22}$ While both have infraredactive phonons, those in $\mathrm{E}$ are vibrons ( $\mathrm{H}-\mathrm{H}$ stretching modes), while those in $\mathrm{D}$ are intermolecular modes. ${ }^{12}$ These results eliminate $\mathrm{B}$ and $\mathrm{D}$, but not $\mathrm{E}$, as candidates for the recently discovered high-pressure phase displaying intense vibron infrared activity. ${ }^{23}$

In summary, we have shown that there are two exceptions to the rule that $s \geq 3$ is a necessary and sufficient minimum-complexity condition for infrared activity in an elemental crystal. One of the two exceptional $s=4$ structures (B of Fig. 1 and Table I) encompasses "wurtzite silicon" and a molecular structure relevant to hydrogen at high pressure.
${ }^{1}$ W. Cochran, Nature (London) 191, 60 (1961); E. Burstein, M. H. Brodsky, and G. Lucovsky, Int. J. Quantum Chem. 1S, 759 (1967); I. Chen and R. Zallen, Phys. Rev. 173, 833 (1968); R. Zallen and G. Lucovsky, in Selenium, edited by R. A. Zingaro and W. C. Cooper (Van Nostrand Reinhold, New York, 1976), p. 148.

${ }^{2}$ R. Zallen, Phys. Rev. 173, 824 (1968).

${ }^{3}$ M. Lax and E. Burstein, Phys. Rev. 97, 39 (1955); M. Lax, Symmetry Principles in Solid State and Molecular Physics (Wiley, New York, 1974), p. 256.

${ }^{4}$ G. Lucovsky, R. C. Keezer, and E. Burstein, Solid State Commun. 5, 439 (1967); R. Geick, U. Schroder, and J. Stuke, Phys. Status Solid 24, 99 (1967).

${ }^{5}$ P. Grosse, M. Lutz, and W. Richter, Solid State Commun. 5, 99 (1967).

${ }^{6}$ R. J. Nemanich, G. Lucovsky, and S. A. Solin, Solid State Commun. 23, 117 (1977).

${ }^{7}$ S. H. Walmsley and A. Anderson, Mol. Phys. 7, 411 (1964); V. Wagner, Phys. Status Solidi B 50, 585 (1972).

${ }^{8}$ R. V. St. Louis and O. Schnepp, J. Chem. Phys. 50, 5177 (1969).
${ }^{9}$ A. Anderson and P. G. Boczar, Chem. Phys. Lett. 43, 506 (1977).

${ }^{10} \mathrm{G}$. Lucovsky, in Physics of Selenium and Tellurium, edited by W. C. Cooper (Pergamon, Oxford, 1969), p. 255.

${ }^{11}$ W. Kratschmer, L. D. Lamb, K. Fostiropoulos, and D. R. Huffman, Nature (London) 347, 354 (1990).

${ }^{12}$ R. M. Martin, V. Natoli, and R. Zallen (unpublished).

${ }^{13}$ Each $s(\alpha)$ is the trace of an $s \times s$ matrix which describes how $\alpha$ permutes the atomic sites; each matrix contains $s$ ones, the other elements are zeros. The quantities $s(\alpha)$ are thus the characters of an $s$-dimensional representation $(S$, the atomicsite representation) of $G$. The sum $\sum s(\alpha)$, taken over the $g$ factor-group operations, is a positive multiple of $g$. To see this, let $A$ denote the fully symmetric representation and $C(S, A)$ be the number of times that $A$ appears in the reduction of $S . C(S, A)$ is given by $(1 / g) \sum s(\alpha) \chi^{A}(\alpha)$. Since $s(1)=s$ and the other $s(\alpha)$ 's are non-negative and each $\chi^{A}(\alpha)=1$, it follows that the sum is positive and that $C(S, A) \geq 1$. $C(S, A)$ has structural significance as the number of distinct sets of equivalent sites contained in the $s$ atoms that comprise the primitive-cell basis. For the structures list- 
ed in Table $\mathrm{I}, C(S, A)$ is 1 for $\mathrm{A}, \mathrm{B}$, and $\mathrm{D}$, and 2 for $\mathrm{C}$ and $\mathrm{E}$.

${ }^{14}$ The notation used here for crystal classes, space groups, and symmetry operations follows that of the International Tables for Crystallography, Volume A, Space-Group Symmetry, edited by T. Hahn (Kluwer, Dordrecht, 1989). Note that $n$ denotes an $n$-fold rotation and $\bar{n}$ an $n$-fold rotation inversion.

${ }^{15}$ In Ref. 2 , p. 830 , column two, the correct results [which include the possibilities $s(6)=0$ and $s(\overline{6})=0]$ are: $6 / m, s=4$, $12 n_{v}^{\text {ir }} \geq 0 ; 6 / \mathrm{mmm}, s=4,24 n_{v}^{\text {ir }} \geq-12$.

${ }^{16}$ In Ref. 2, the possible $s=4$ structures for the ten $m 3 m$ cubic space groups were examined in a similar way. It is interesting that it is not these highest-symmetry $(g=48)$ groups, but instead the $6 / \mathrm{mmm}(g=24)$ groups, which harbor the exceptions to (2).

${ }^{17}$ See the discussion following Eq. (7.4) in Ref. 2.
${ }^{18} \mathrm{~J}$. Donohue, The Structures of the Elements (Wiley, New York, 1974).

19"Wurtzite silicon" is actually a misnomer for Lonsdaleite. The $P 6_{3} / \mathrm{mmc}$ space group of Lonsdaleite has twice the symmetry of the $P 6_{3} m c$ space group of wurtzite (just as diamond has twice the symmetry of zinc blende).

${ }^{20}$ R. H. Wentorf and J. S. Kasper, Science 139, 338 (1963).

${ }^{21}$ C. Frondel and U. B. Marvin, Nature (London) 214, 587 (1967); F. P. Bundy and J. S. Kasper, J. Chem. Phys. 46, 3437 (1967).

${ }^{22}$ H. Nagara and T. Nakamura, Phys. Rev. Lett. 68, 2468 (1992). $E$ is shown in Fig. 1(d).

${ }^{23}$ M. Hanfland, R. J. Hemley, and H. K. Mao, Phys. Rev. Lett. 70, 3760 (1993). 